\title{
一种新型膦酸锆孔材料的合成及其对金属离子的吸附性质
}

\author{
Abubaker Abutartour, Lotfia El Majdoub，史亚赛，李妮丽，徐庆红 \\ (北京化工大学化工资源有效利用国家重点实验室, 北京 100029)
}

摘 要: 骨架含强配位基的有机无机杂化多级孔材料合成是目前吸附研究的一个重要领域。以 bis (hexamethylene) triamino- $\mathrm{N}, \mathrm{N}$-bisacetyl-phosphonic acid 和 $\mathrm{ZrOCl}_{2} \cdot 8 \mathrm{H}_{2} \mathrm{O}$ 为原料、应用水热合成技术合成了一种新型膦酸铅多级孔材 料 ZrPTA，并通过 FT-IR、TGA、XRD、XPS、SEM 以及元素分析等手段对制备产物进行了表征。研究结果表明, ZrPTA 具有棒状微形貌, 其内部存在大量直径为 $1.38 \mathrm{~nm}$ 和 $1.93 \mathrm{~nm}$ 的微孔以及直径为 $2.99 \mathrm{~nm}$ 的介孔, 其表面积为 $112.2 \mathrm{~m}^{2} / \mathrm{g}$ 。 ZrPTA 对水溶液中 $\mathrm{Pb}^{2+} 、 \mathrm{Cu}^{2+}$ 和 $\mathrm{Cd}^{2+}$ 三种金属离子具有良好的吸附作用，最大吸附量分别为 742.7、689.8 和 $627.0 \mathrm{mg} / \mathrm{g}$, 远远高于文献报道值。这一性质使 ZrPTA 具有潜在的废水处理功效，显示出诱人的应用前景。

关 键 词: 膦酸锆; 孔材料; 水热合成; 内表面; 吸附

中图分类号: TQ174 文献标识码: A

\section{A New Porous Zirconium Phosphonate Hybride Material and Its Adsorption Properties}

\author{
ABUBAKER Abutartour, LOTFIA El-Majdoub, SHI Ya-Sai, LI Ni-Li, XU Qing-Hong
}

(State Key Laboratory of Chemical Resource Engineering, Beijing University of Chemical Technology, Beijing 100029, China)

\begin{abstract}
Synthesis of organic-inorganic hybrid hierarchical porous materials with strong ligand in framework is an important field of current research in adsorption. A new kind of inorganic/organic hierarchical zirconium phosphonate material (ZrPTA) with porous structure was prepared from bis(hexamethylene)triamino-N, N-bisacetylphosphonic acid and $\mathrm{ZrOCl}_{2} \cdot 8 \mathrm{H}_{2} \mathrm{O}$ in water solution by using hydrothermal synthesis technology. The samples were characterized by FT-IR spectroscope, TGA, XRD, XPS, SEM and element analysis. The results show that the material possesses a rod-like morphology, and the rods contain micropores of $1.38 \mathrm{~nm}$ and $1.93 \mathrm{~nm}$ in diameter, mesopores of $2.99 \mathrm{~nm}$ in diameter, and a surface area of $\sim 112.2 \mathrm{~m}^{2} / \mathrm{g}$. The as-prepared ZrPTA is more efficient in removing $\mathrm{Pb}^{2+}, \mathrm{Cu}^{2+}$ and $\mathrm{Cd}^{2+}$ from wastewater. Its maximum adsorption capacities for $\mathrm{Pb}^{2+}, \mathrm{Cu}^{2+}$ and $\mathrm{Cd}^{2+}$ are $742.7,689.8$ and $627.0 \mathrm{mg} / \mathrm{g}$, respectively, which is much higher than the maximum adsorption capacities of the adsorbents reported. The perfect properties make the material good prospects in application as an adsorbent in wastewater processing.
\end{abstract}

Key words: zirconium phosphonate; porous materials; hydrothermal synthesis; interfaces; adsorption

近年来, 有关材料的吸附和分离功能的研究 已经成为一个研究热点, 大量高效吸附剂被合成 出来 ${ }^{[1-3]}$ 。一些杂化孔材料具有良好的离子吸附性
能，它们能够从废水中去除 $\mathrm{Cd}^{2+} 、 \mathrm{Cu}^{2+}$ 和 $\mathrm{Pb}^{2+}$ 等重 金属离子，而这些重金属离子被公认具有强烈的环 境毒害作用 ${ }^{[4]}$ 。这些孔状吸附剂的孔内有机官能团 
能够与金属离子配位, 在重金属离子去除中扮演着 重要角色 ${ }^{[5-6]}$ 。

近年来合成了一系列高吸附性能材料, 例如金 属氧化物孔材料 ${ }^{[7]}$ 、磷酸盐孔材料 ${ }^{[8]}$ 以及有机膦酸 盐孔材料 ${ }^{[9]}$ 等。2008 年, Zhang 等 ${ }^{[10]}$ 利用自组装技术 成功地合成了有机膦酸钛杂化孔材料, 该材料对水 溶液中重金属离子具有惊人的吸附容量。

$\mathrm{Zr}\left(\mathrm{HPO}_{4}\right)_{2} \cdot \mathrm{H}_{2} \mathrm{O}(\alpha-\mathrm{ZrP})$ 及其衍生物是一类重要 的层状无机材料 ${ }^{[11-12]}$, 一直倍受化学及材料学界 的重视。 $\alpha-\mathrm{ZrP}$ 系列衍生物之一的有机膦酸锆 $\mathrm{Zr}\left(\mathrm{O}_{3} \mathrm{PR}\right)_{2}(\mathrm{R}$ 为链接于磷酸锆层壁上有机基团) 自 1978 年被合成以来 ${ }^{[13]}$ ，由于其骨架结构中 $\mathrm{R}$ 基团的 可修饰性, 使得此类有机/无机复合材料具有层内空 间的变化设计性。膦酸锆在催化 ${ }^{[14]}$ 、离子交换 ${ }^{[15]}$ 、 吸附 ${ }^{[12,16]}$ 以及其它方面 ${ }^{[17-18]}$ 得到了广泛研究。在表 面活性剂的辅助下, 采用溶胶一凝胶合成手段, 有机 分子插层进而得到柱撑磷酸锆孔状材料 ${ }^{[13,17-18]}$ 得到 了迅猛发展。膦酸铅孔材料由于具有良好的热稳定 性、可观的比表面积、酸性可调节性以及孔大小的 可调变性, 在催化、稀土配位发光、金属离子吸附 等诸多领域显示出诱人的应用前景。

本工作报道一种新型有机膦酸锆杂化孔材料 ZrPTA 的合成及其对金属离子的吸附性能, 并考察 $\mathrm{ZrPTA}$ 对水溶液中 $\mathrm{Pb}^{2+} 、 \mathrm{Cu}^{2+}$ 和 $\mathrm{Cd}^{2+}$ 离子的吸附去 除效果。

\section{1 实验方法}

\section{1 实验仪器和试剂}

主要仪器: Rigaku D/MAX X 射线衍射仪, Bruker Vector-22 红外光谱分析仪, Perkin-Elmer plasma 40 emission 感应耦合等离子体分析仪, Vario-EL 元素分析仪, Shimadzu SS-550 型扫描电子显 微镜, JEOL JEM-1000 透射电子显微镜, Quantachrome NOVA 2000e 吸附仪, HCT-2 热分析仪, Shimadzu ESCA-750 和 ESCA-1000 X 光电子能谱仪, Agilent 4510 原子吸收风光光度计。利用 BrunauerEmmett-Teller(BET) 计算法得到样品的比表面积, 通过 Barret-Joyner-Halenda(BJH) 分析法得到样品的 孔分布。

主要试剂: 八水合氧氯化锆 $(>98 \%$, 分析纯)、 双(六甲撑)三胺 $[$ Bis(hexamethylene)triamine $](>99 \%$, 分析纯)分别购自于 Sinopharm 试剂公司(上海)及 Sigma-Aldrich 试剂公司(美国); 三氯氧磷(分析纯)、 硝酸铅(分析纯)、硝酸铜(分析纯)以及硝酸镉(分析
纯)系北京化学试剂公司产品。

\section{2 化合物 bis(hexamethylene)triamino-N-N- bisacetyl-phosphorus oxychloride 的合成}

在氮气保护下, 将 $3.59 \mathrm{~g}$ 双(六甲撑)三胺溶于 $50 \mathrm{~mL}$ 干燥甲苯中, 再将上述甲苯溶液加入到干燥 的、充满氮气的圆底烧瓶中(烧瓶一直处于冰水浴冷 却之下)。然后向冰水浴冷却下的烧瓶体系中缓慢滴 加三氯氧磷甲苯溶液 $(4.73 \mathrm{~mL}$ 三氯氧磷溶于 $20 \mathrm{~mL}$ 甲苯中)。滴加完毕后, 混合物在 $0^{\circ} \mathrm{C}$ 下搅拌并保温 $3 \mathrm{~h}$, 然后缓慢升至室温。室温下，向上述体系中滴 加 $2.5 \mathrm{~mL}$ 乙酰氯, 并搅拌 $5 \mathrm{~h}$ 。反应结束后, 经离心 分离所得固体物质用干燥甲苯洗涤数次, 并在 $100^{\circ} \mathrm{C}$ 干燥。以上所有步骤均在氮气保护条件下进行。

\section{3 有机膦酸锆孔材料 ZrPTA 的合成}

将 3 滴 $40 \%$ 的氢氟酸溶液滴加到 $\mathrm{Zr}^{4+}$ 水溶液中 $\left(5.4 \mathrm{~g} \mathrm{ZrOCl}_{2} \cdot 8 \mathrm{H}_{2} \mathrm{O}\right.$ 溶于 $20 \mathrm{~mL}$ 去离子水中)。在剧烈摚 拌下, 将上述 $\mathrm{Zr}^{4+}$ 溶液滴加到合成的 bis(hexamethylene) triamino-N-N-bisacetylphosphoru-s oxychloride 水溶 液中, 直至形成白色悬浊液。混合体系在搅拌下 $120^{\circ} \mathrm{C}$ 回流 $24 \mathrm{~h}$ 后转移至内祄聚四氟乙烯的不锈钢 反应釜中, $80^{\circ} \mathrm{C}$ 晶化 $2 \mathrm{~d}$, 体系经离心分离, 所得固 体物质用去离子水洗涤数次, 并在 $70^{\circ} \mathrm{C}$ 干燥, 得到 产物 ZrPTA。

\section{4 金属离子吸附测试}

称取 $0.10 \mathrm{~g} \mathrm{ZrPTA}$ 数份, 分别加入到 $100 \mathrm{~mL}$ 不 同浓度 $\mathrm{Pb}\left(\mathrm{NO}_{3}\right)_{2} 、 \mathrm{Cd}\left(\mathrm{NO}_{3}\right)_{2}$ 和 $\mathrm{Cu}\left(\mathrm{NO}_{3}\right)_{2}$ 的水溶液 中。混合物在室温下 $\left(25 \pm 2^{\circ} \mathrm{C}\right)$ 搅拌 $24 \mathrm{~h}$ 以确保达 到吸附平衡。汲取固液混合物经离心分离后的上层 清液，利用原子吸收法测定清液中金属离子的浓 度。利用式(1) ${ }^{[19]}$ 计算吸附剂的吸附容量:

$$
q_{\mathrm{e}}=\left(c_{0}-c_{\mathrm{e}}\right) V / W
$$

式中: $q_{\mathrm{e}}$ 为单位质量吸附剂所吸附金属离子的质量, 单位为 $\mathrm{mg} / \mathrm{g} ; c_{0}$ 和 $c_{\mathrm{e}}$ 分别为溶液中金属离子被吸附 前后浓度, 单位为 $\mathrm{mg} / \mathrm{L} ; V$ 是金属离子溶液的体积; $W$ 为吸附剂的质量。

\section{2 结果与讨论}

\section{1 材料的制备与表征}

利用双(六甲撑)三胺、三氯氧磷和乙酰氯反应制备 Bis(hexamethylene)triamino-N-N-bisacetyl-phosphorus oxychloride。产物的 ${ }^{1} \mathrm{H} \mathrm{NMR}\left(400 \mathrm{MHz}, \mathrm{CDCl}_{3}\right)$ 化学 位移 $(\delta)$ 为 $1.2\left(\mathrm{CH}_{3}, 6 \mathrm{H}, \mathrm{t}\right), 1.5-2\left(\mathrm{CH}_{2}, 24 \mathrm{H}, \mathrm{m}\right) ;{ }^{13} \mathrm{C}$ $\operatorname{NMR}\left(400 \mathrm{MHz}, \mathrm{CDCl}_{3}\right)$ 化学位移 $(\delta)$ 为 $18.2\left(\mathrm{CH}_{3}\right), 23.6$ 、 
$25 、 28.2$ 和 $33.7\left(12 \mathrm{CH}_{2}\right) ; 40.2\left(\mathrm{~N}-\mathrm{CH}_{2}\right), 159.1(\mathrm{C}=\mathrm{O})$ 。 以上数据初步表明, 合成产物为目标产物且纯度较 高, 并且产物中羧基的存在以及 $\mathrm{H}$ 原子个数与分子 结构中 $\mathrm{H}$ 原子个数的吻合。化合物 bis (hexamethylene)triamino-N-N-bisacetylphosphorus oxychloride 分子结构如图 1 所示。图 2A 显示了双 (六甲撑)三胺(a)和 bis(hexamethylene)triamino-N-Nbisacetyl-phosphorus oxychloride(b)的红外光谱图。 $\mathrm{N}-\mathrm{H} 、 \mathrm{CH}_{2}$ 中的 $\mathrm{C}-\mathrm{H}$ 红外吸收在两个化合物的谱图 中分别位于 3277 和 $2931 \mathrm{~cm}^{-1}$ 处。图 $2 \mathrm{~A}(\mathrm{~b})$ 中 N-P $\left(732 \mathrm{~cm}^{-1}\right), \mathrm{P}-\mathrm{Cl}\left(1521 \mathrm{~cm}^{-1}\right)$ 以及 $\mathrm{P}=\mathrm{O}\left(1265 \mathrm{~cm}^{-1}\right)$ 键红外吸收的出现证明双(六甲撑)三胺和三氯氧磷 之间发生了化学反应, 这些化学键的红外吸收在图 2A(a)中并不存在。

$\mathrm{ZrPTA}$ 是在 bis(hexamethylene)triamino-N-N- bisacetyl-phosphorus oxychloride、 $\mathrm{Zr}^{4+}$ 以及氢氟酸参与下 合成得到的。红外光谱(图 $2 \mathrm{~A}(\mathrm{c})$ ) 显示, 原反应物 bis(hexamethylene)triamino-N-N-bisacetyl-phosphorus oxychloride 中 $\mathrm{P}-\mathrm{Cl}$ 吸收消失, 说明该化合物已经转化 为其相应的有机酸, 并参与了 ZrPTA 的合成反应。

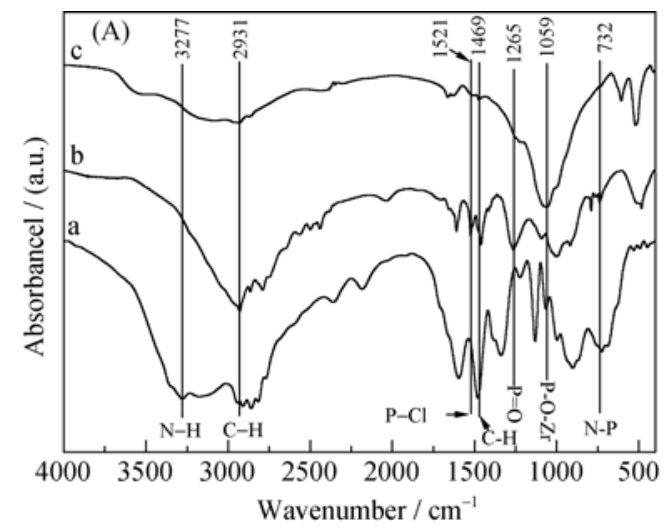<smiles>CC(=O)N(C)P(=O)(Cl)Cl</smiles>

图 1 Bis(hexamethylene)triamino-N-N-bisacetyl-phosphorus 分 子结构

Fig. 1 Structures of bis(hexamethylene)triamino-N-N-bisacetylphosphorus oxychloride

位于 $1059 \mathrm{~cm}^{-1}$ 波数处、属于 $\mathrm{P}-\mathrm{O}-\mathrm{Zr}$ 键且较为尖锐的 吸收峰出现, 初步证明 ZrPTA 骨架结构已经形成 ${ }^{[20-22]}$ 。 另外，在产物 ZrPTA 的吸收光谱中发现存在 N-P 键 红外吸收, 说明产物形成的水解过程没有破坏有机 膦酸分子结构。

图 3 是产物 ZrPTA 在 $3^{\circ} \sim 70^{\circ}$ 区间的 PXRD 图 谱。在 $10.26^{\circ}(002) 、 20.60^{\circ}(100) 、 25.82^{\circ}(112)$ 处有 三个主衍射峰，对应的晶面间距分别是 $0.861 、 0.431$ 和 $0.345 \mathrm{~nm}$ 。三个衍射峰的出现表明 ZrPTA 具备磷 酸锆类材料的基本骨架特征。图谱中 $2 \theta=6.41^{\circ}$ 处出 现了一衍射峰, 对应的材料微孔直径为 $1.38 \mathrm{~nm}$ 。孔

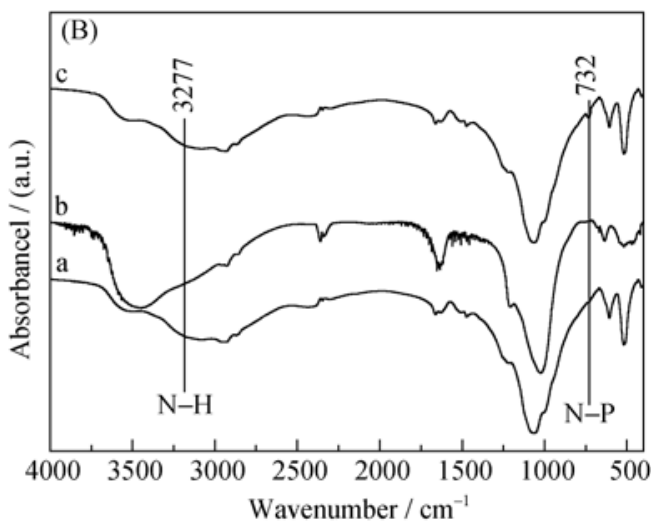

图 2 (A)双(六甲撑)三胺(a)、bis(hexamethylene)triamino-N-N-bisacetylphosphorus oxychloride(b)及 ZrPTA(c)的红外光谱图;

(B) ZrPTA 经过不同 $\mathrm{pH}$ 盐酸溶液浸泡 $1 \mathrm{~h}$ 后样品的红外光谱 $(\mathrm{a}: \mathrm{pH}=5.0 ; \mathrm{b}: \mathrm{pH}=6.0 ; \mathrm{c}$ : 未浸泡)

Fig. 2 (A) FT-IR spectra of bis(hexamethylene)triamine (a), bis(hexamethylene)triamino-N-N-bisacetyl-phosphorus oxychloride (b) and ZrPTA (c); (B) FT-IR spectra of ZrPTA immersed in $\mathrm{HCl}$ solutions with different $\mathrm{pH}$ (a: $\mathrm{pH}=5.0$; b: $\mathrm{pH}=6.0$; $\mathrm{c}$ : unimmersed)
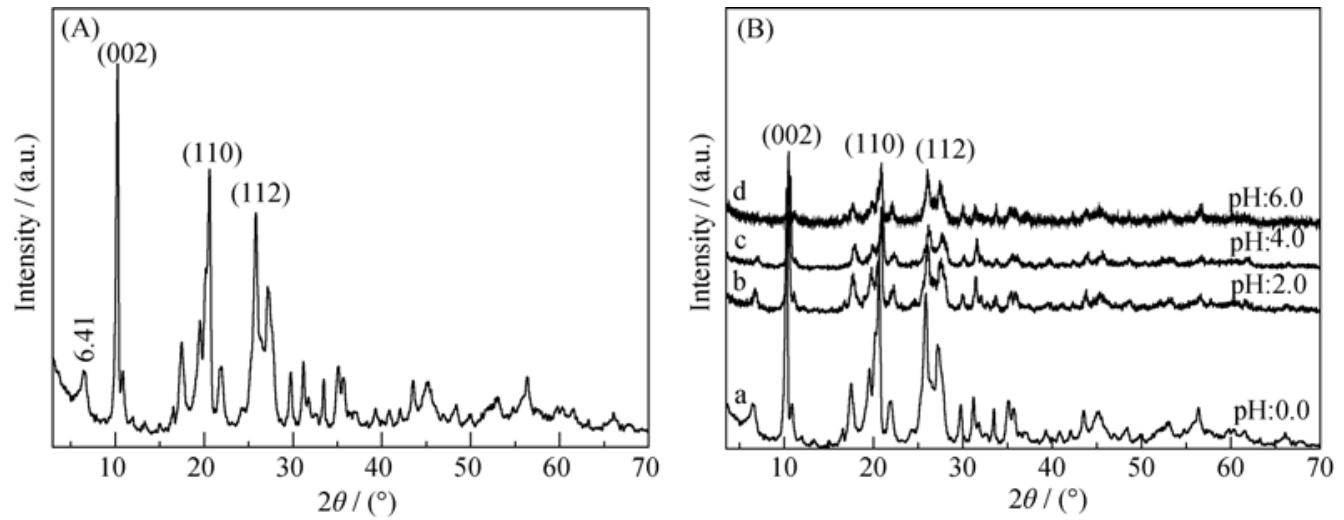

图 $3 \mathrm{ZrPTA}(\mathrm{A})$ 及经过不同 $\mathrm{pH}$ 盐酸溶液处理后的 $\mathrm{ZrPTA}(\mathrm{B})$ 的 XRD 图谱

Fig. 3 PXRD patterns of ZrPTA (A) and ZrPTA delt in $\mathrm{HCl}$ solutions with different $\mathrm{pH}$ (B) 

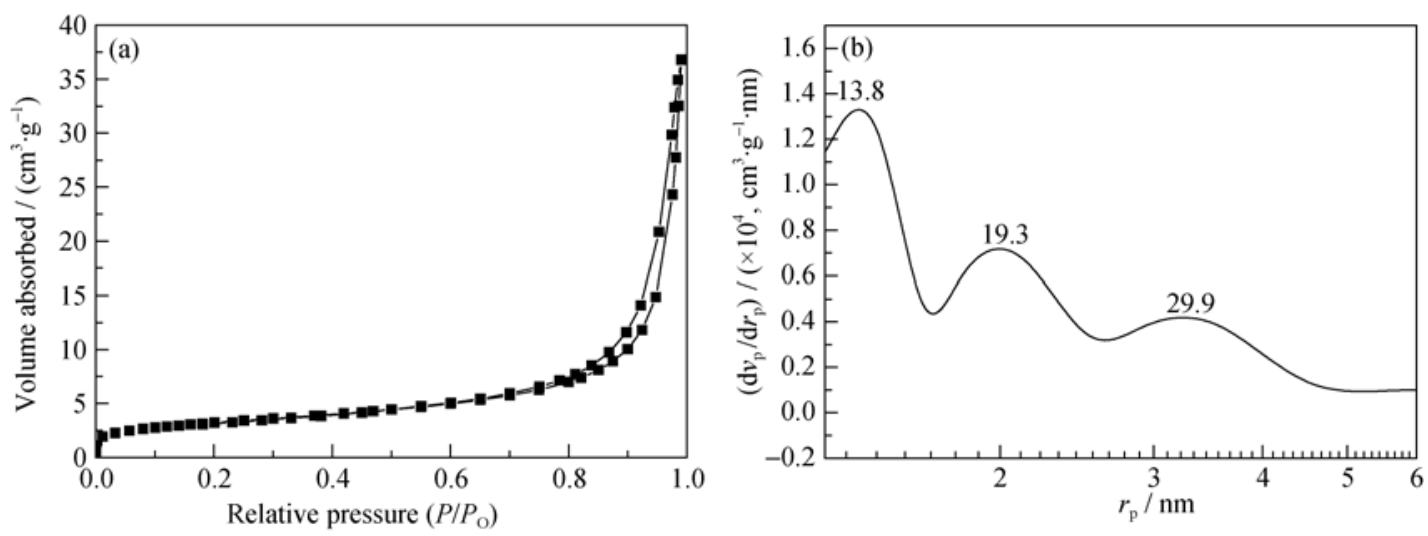

图 $4 \mathrm{ZrPTA}$ 的氮气吸脱附曲线(a)及孔分布曲线(b)

Fig. $4 \mathrm{~N}_{2}$ adsorption-desorption isotherms curve (a) and pore width distribution (b) of ZrPTA

分布(图 4b)分析显示, ZrPTA 内部除了存在直径为 1.38 和 $1.93 \mathrm{~nm}$ 微孔, 还存在直径为 $2.99 \mathrm{~nm}$ 的介 孔。ZrPTA 氮气吸脱附等温线如图 4(a)所示。BJH 和 BET 模式计算表明, ZrPTA 是一种兼具微孔和介孔的 孔材料, 其内部大部分孔直径分布在 $1.38 \mathrm{~nm}$ 左右。 由于 $\mathrm{ZrPTA}$ 的三个特征衍射峰强度较强, 且直径为 $1.38 \mathrm{~nm}$ 的微孔在材料内部占据主导地位, 故直径为 $1.93 \mathrm{~nm}$ 和 $2.99 \mathrm{~nm}$ 介孔信息在图 3 中没有体现出来。

合成材料中存在的三种不同孔径结构的孔可能 是由于不同膦酸分子中不同位置磷羧基与 $\mathrm{Zr}^{4+}$ 反应 所形成的。如图 5 所示, 数个膦酸中的 $1 、 3$ 号位磷 羧基与 $\mathrm{Zr}^{4+}$ 反应, 彼此相互链接形成直径为 $2.99 \mathrm{~nm}$ 的介孔; 一个膦酸分子中的 $1 、 2$ (或 $2 、 3$ ) 号位磷羧 基与其它膦酸分子中的 $1 、 2$ 号位磷翔基通过 $\mathrm{O}-\mathrm{Zr}-\mathrm{O}$ 键链接(其中存在数个膦酸分子参与反应)形成 $1.93 \mathrm{~nm}$ 的微孔; 而直径为 $1.38 \mathrm{~nm}$ 的微孔则可能是由膦酸 分子中的 1(或 3)、2 号位磷羧基与其它膦酸分子中 的 3(或 1)、2 号位磷羧基通过 $\mathrm{O}-\mathrm{Zr}-\mathrm{O}$ 键链(其中存 在数个膦酸分子参与反应) 而形成的。

从图 6(a)给出的 ZrPTA 透射电镜照片可以看出,

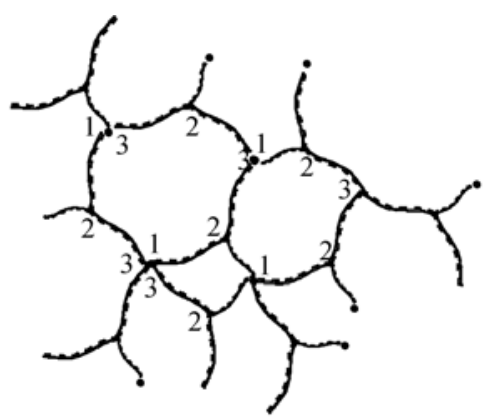

$2^{3}\left(\begin{array}{l}\text { : one molecular union of bis(hexamethylene)triamino- } \\ \text { N-N-bisacetyl-phosphorus acid }\end{array}\right.$
- : $\mathrm{Zr}^{4+}$

图 $5 \mathrm{ZrPTA}$ 可能的孔结构示意图

Fig. 5 Possible porous structure in ZrPTA
材料内部存在微、介孔结构, 但由于结晶度较低, 在合 成材料内部不能够明显地区分。BET 法计算结果表明, ZrPTA 的比表面积和比孔容分别为 $112.2 \mathrm{~m}^{2} / \mathrm{g}$ 和 $0.6 \mathrm{~cm}^{3} / \mathrm{g}$ 左右。扫描电镜照片(图 6(b))显示, ZrPTA 的形貌呈长 条片状, 其平均长度和宽度为 $1.2 \mu \mathrm{m}$ 和 $0.15 \mu \mathrm{m}$ 左右。

热稳定性测试(如图 7 所示)结果显示, ZrPTA 有三 个失重区间。第一个失重区间发生在室温至 $158^{\circ} \mathrm{C}$ 之 间, 大约有 $2.52 \%$ 的质量失去, 该失重过程对应的吸 热峰出现在 $115^{\circ} \mathrm{C}$ 左右。这一失重过程可以归结为材 料表面吸附水及材料内部结构水失去。第二个失重过 程发生在 $158 \sim 268^{\circ} \mathrm{C}$ 之间, 这一过程大约有 $1.15 \%$ 的 质量失去, 所失去的质量主要来自于 ZrPTA 内外表面 所吸附的有机物。第三个失重过程发生在 $268 \sim 616^{\circ} \mathrm{C}$ 之间，此过程大约有 $12.26 \%$ 的质量失去, 该过程主要 表现为放热过程, 三个主要放热峰分别位于 $358^{\circ} \mathrm{C}$ 、

$432^{\circ} \mathrm{C}$ 和 $501{ }^{\circ} \mathrm{C}$, 此过程质量失去主要来自于 $\mathrm{ZrPTA}$ 骨架结构中有机链的燃烧并彻底以二氧化碳和水形 式的放出。由此可见, $\mathrm{ZrPTA}$ 在 $268^{\circ} \mathrm{C}$ 以下温度环境中 是稳定的。元素含量分析结果显示, ZrPTA 中 Zr、O、 $\mathrm{P} 、 \mathrm{C} 、 \mathrm{H}$ 及 $\mathrm{N}$ 的百分含量分别为 $60.79 、 14.96 、 6.21$ 、 $12.83 、 2.41$ 及 $2.80 \%$, 其基本分子结构为 $\mathrm{Zr}_{10}\left(\mathrm{C}_{16} \mathrm{H}_{30} \mathrm{O}_{11} \mathrm{~N}_{3} \mathrm{P}_{3}\right) \cdot 3 \mathrm{H}_{2} \mathrm{O}$ 。

实验对 ZrPTA 的酸碱稳定性也进行了研究。经 过不同浓度盐酸溶液浸泡后 ZrPTA 的 PXRD 衍射图 谱如图 3(B)所示。结果显示, 当盐酸溶液的 $\mathrm{pH}>4.0$, $\mathrm{ZrPTA}$ 的骨架结构开始发生分解; $\mathrm{ZrPTA}$ 在 $\mathrm{pH} \leq 4.0$ 的酸性溶液中能够稳定存在。红外光谱也证明了上 述结论。当 $\mathrm{ZrPTA}$ 浸泡于 $\mathrm{pH}=5.0$ 盐酸溶液 $1 \mathrm{~h}$ 后, 所 得固体物质中的 $\mathrm{N}-\mathrm{P}$ 及 $\mathrm{N}-\mathrm{H}$ 键吸收强度与 $\mathrm{ZrPTA}$ 相比大大降低; 而用盐酸溶液 $\mathrm{pH}=6.0$ 浸泡 $1 \mathrm{~h}$ 后, 固体样品中 $\mathrm{N}-\mathrm{P}$ 及 $\mathrm{N}-\mathrm{H}$ 键红外吸收几乎消失, 说明 样品骨架已经解体(图 2B)。 

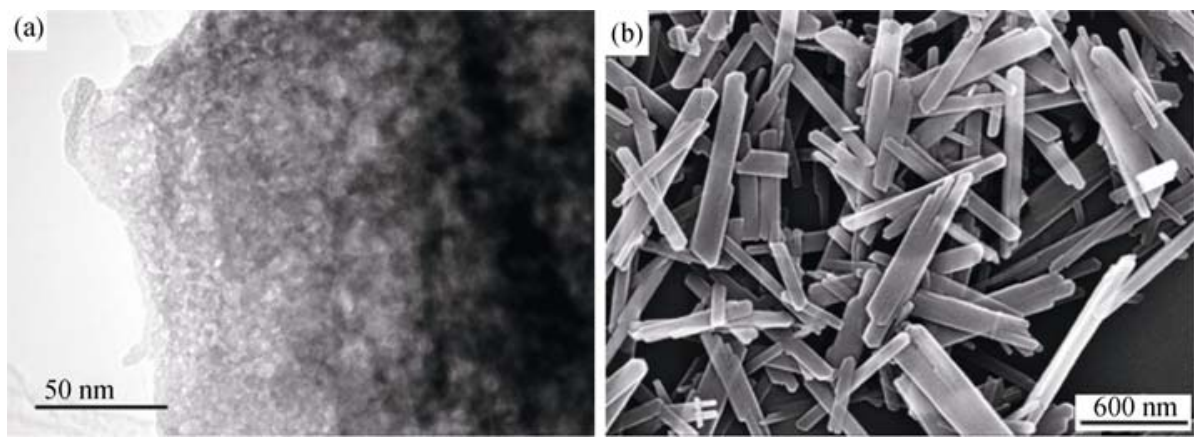

图 $6 \mathrm{ZrPTA}$ 的透射电镜(a)及扫描电镜(b)照片

Fig. 6 TEM (a) and SEM (b) images of ZrPTA

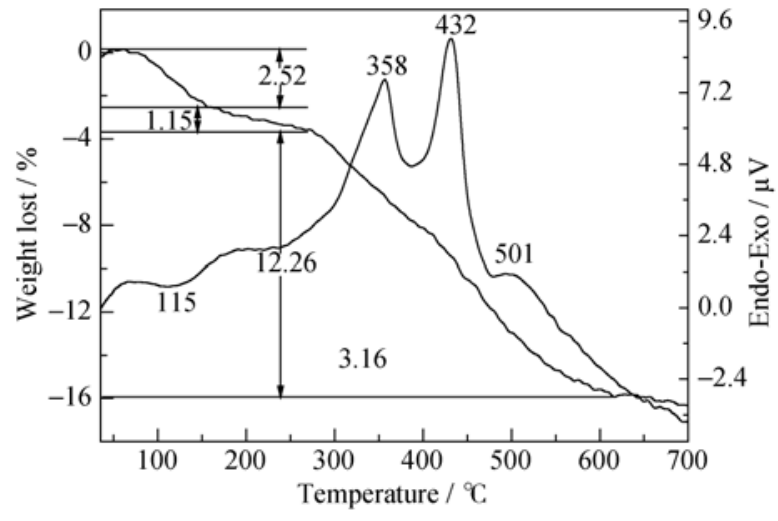

图 $7 \mathrm{ZrPTA}$ 的差热-热失重分析曲线

Fig. 7 TG-DTA curves of ZrPTA

\subsection{ZrPTA 对金属离子的吸附行为}

$\mathrm{ZrPTA}$ 在水溶液中对 $\mathrm{Pb}^{2+} 、 \mathrm{Cu}^{2+}$ 和 $\mathrm{Cd}^{2+}$ 离子的
吸附行为进行了深入研究。将一定质量的 ZrPTA 分 别加入到含 $\mathrm{Pb}^{2+} 、 \mathrm{Cu}^{2+}$ 和 $\mathrm{Cd}^{2+}$ 离子的水溶液中, 并保 持金属离子水溶液 $\mathrm{pH}$ 为 4.0 。室温下, $\mathrm{ZrPTA}$ 对不同 浓度的 $\mathrm{Pb}^{2+} 、 \mathrm{Cu}^{2+}$ 和 $\mathrm{Cd}^{2+}$ 离子的吸附曲线如图 8 所示。 由图 8 可知，溶液中金属离子浓度随吸附时间的增加 而逐渐下降，当吸附时间超过 $800 \mathrm{~min}$ 后，吸附行为 趋于平衡。计算结果显示, 当金属离子的起始浓度为 $900 \mathrm{mg} / \mathrm{L}$ 时，吸附剂对上述三种金属离子的最大吸附 容量 $\left(q_{\mathrm{e}}, \mathrm{mg} / \mathrm{g}\right)$ 分别为 742.96 (对 $\mathrm{Pb}^{2+}$ )、689.81 (对 $\mathrm{Cu}^{2+}$ ) 和 627.04 (对 $\mathrm{Cd}^{2+}$ ) (相关数据详见表 1)。研究者 将 ZrPTA 对上述三种金属离子的最大吸附容量与文 献[20-22]报道的有关吸附剂对三种离子最大吸附容 量进行了比较(见表 2)。结果发现, ZrPTA 对上述三种 金属离子的最大吸附量大于文献所报道值。
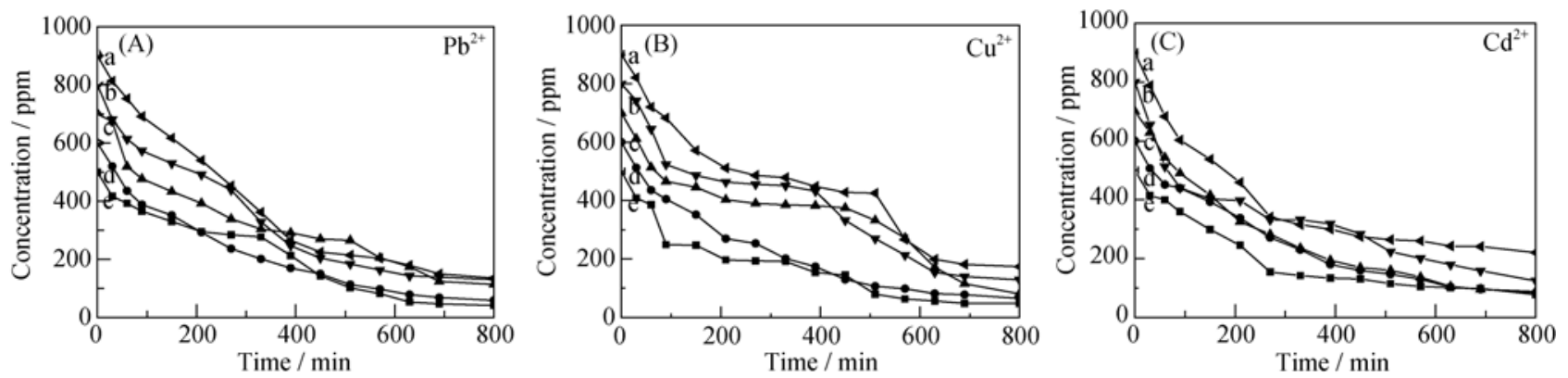

图 $8 \mathrm{ZrPTA}$ 在 $\mathrm{pH}=4.0$ 的酸性环境中对不同浓度金属离子的吸附随时间变化关系曲线

Fig. 8 Change of adsorptions of ZrPTA to metal ions with different concentrations with time (adsorbent dosage $=1.0 \mathrm{~g} / \mathrm{L}$, temperature is $(25 \pm 2)^{\circ} \mathrm{C}, \mathrm{pH}=4.0$ )

(a) $900 \mathrm{mg} / \mathrm{L}$; (b) $800 \mathrm{mg} / \mathrm{L}$; (c) $700 \mathrm{mg} / \mathrm{L}$; (d) $600 \mathrm{mg} / \mathrm{L}$; (e) $500 \mathrm{mg} / \mathrm{L}$

表 1 吸附剂 ZrPTA 对三种金属离子在不同起始浓度条件下的吸附量 $(\mathrm{mg} / \mathrm{g})$ 及吸附百分率 Table 1 Adsorption date (mg/g) and adsorption percents (\%) (in the bracket) of the adsorbent to three metal ions at different origin concentrations

\begin{tabular}{lccccc}
\hline & $500 /\left(\mathrm{mg} \cdot \mathrm{L}^{-1}\right)$ & $600 /\left(\mathrm{mg} \cdot \mathrm{L}^{-1}\right)$ & $700 /\left(\mathrm{mg} \cdot \mathrm{L}^{-1}\right)$ & $800 /\left(\mathrm{mg} \cdot \mathrm{L}^{-1}\right)$ & $900 /\left(\mathrm{mg} \cdot \mathrm{L}^{-1}\right)$ \\
\hline $\mathrm{Pb}^{2+}$ & $459.21(91.84)$ & $541.25(90.20)$ & $586.33(83.76)$ & $670.15(83.76)$ & $742.96(82.55)$ \\
$\mathrm{Cd}^{2+}$ & $422.98(84.59)$ & $516.33(86.05)$ & $611.97(87.42)$ & $676.21(84.52)$ & $627.04(69.67)$ \\
$\mathrm{Cu}^{2+}$ & $452.15(90.43)$ & $534.87(89.14)$ & $619.43(88.48)$ & $671.04(83.88)$ & $689.81(76.64)$ \\
\hline
\end{tabular}


表 2 ZrPTA 对 $\mathbf{P b}^{2+} 、 \mathrm{Cu}^{2+}$ 及 $\mathbf{C d}^{2+}$ 的最大吸附量与来自不同文献吸附剂最大吸附量的比较

Table 2 Comparison of qe of $\mathrm{ZrPTA}$ for $\mathrm{Pb}^{2+}, \mathrm{Cu}^{2+}$ and $\mathrm{Cd}^{2+}$ with those of different types of adsorbents in references

\begin{tabular}{|c|c|c|c|c|c|c|c|c|c|}
\hline Adsorbents & $\begin{array}{l}\text { Magnetic che- } \\
\text { lating resin }\end{array}$ & $\begin{array}{c}\text { Zwitterionic } \\
\text { hybrid polymers }\end{array}$ & ZrPTA & $\begin{array}{c}\text { Polycar- } \\
\text { boxy-lated starch }\end{array}$ & $\begin{array}{l}\text { Ion-impri } \\
\text { nted fiber }\end{array}$ & ZrPTA & $\begin{array}{c}\text { Porous } \\
\text { bioadsorbent }\end{array}$ & $\begin{array}{l}\text { Activated } \\
\text { carbon }\end{array}$ & ZrPTA \\
\hline & & to $\mathrm{Pb}^{2+}$ & & & to $\mathrm{Cu}^{2+}$ & & & to $\mathrm{Cd}^{2+}$ & \\
\hline$q_{\mathrm{e}} /\left(\mathrm{mg} \cdot \mathrm{g}^{-1}\right)$ & 571.8 & 380.2 & 742.7 & 128.3 & 120.0 & 689.8 & 278.6 & 157.4 & 627.0 \\
\hline Temperature & $45^{\circ} \mathrm{C}$ & $45^{\circ} \mathrm{C}$ & $25^{\circ} \mathrm{C}$ & $40^{\circ} \mathrm{C}$ & $30^{\circ} \mathrm{C}$ & $25^{\circ} \mathrm{C}$ & $25^{\circ} \mathrm{C}$ & $25^{\circ} \mathrm{C}$ & $25^{\circ} \mathrm{C}$ \\
\hline $\mathrm{pH}$ & 6.0 & 5.0 & 4.0 & 7.0 & 6.0 & 4.0 & 5.0 & 5.0 & 4.0 \\
\hline Ref & & [20] & & & 21] & & & [22] & \\
\hline
\end{tabular}

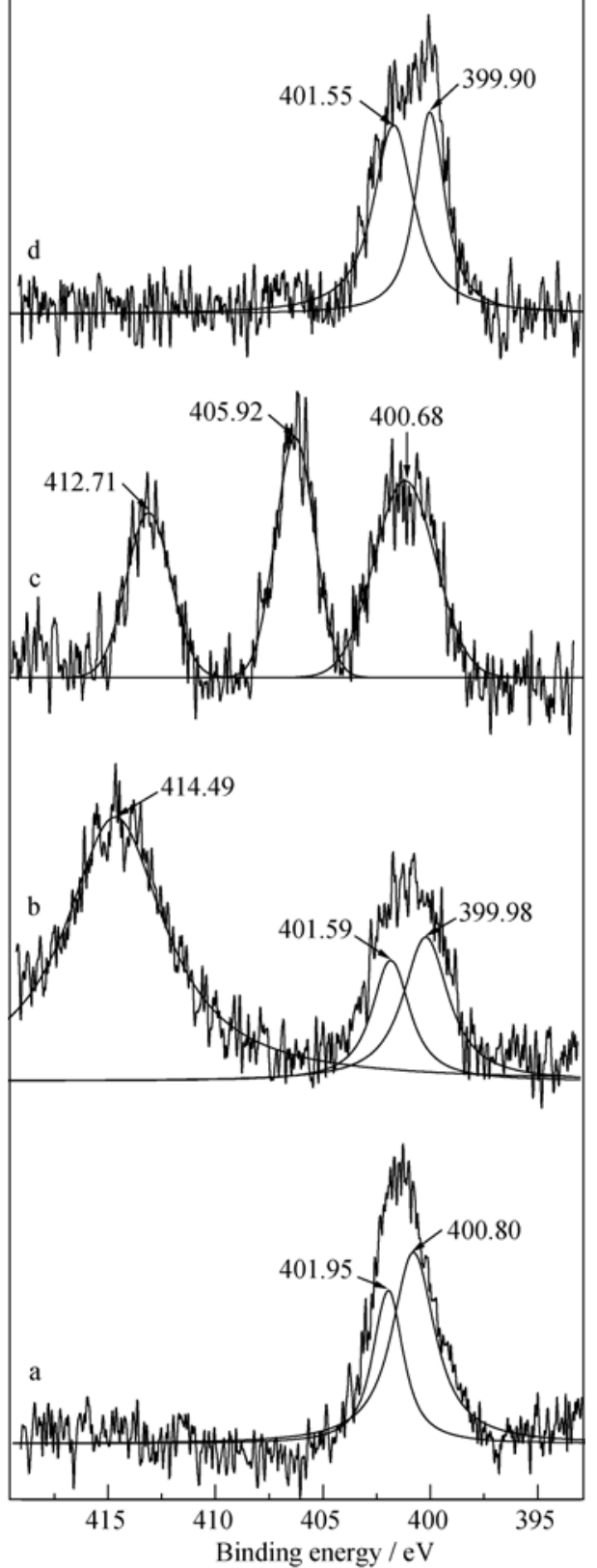

图 $9 \mathrm{ZrPTA}(\mathrm{a}) 、 \mathrm{ZrPTA} / \mathrm{Pb}^{2+}(\mathrm{b}) 、 \mathrm{ZrPTA} / \mathrm{Cd}^{2+}(\mathrm{c})$ 及 $\mathrm{ZrPTA} / \mathrm{Cu}^{2+}(\mathrm{d})$ 中 $\mathrm{N} 1 \mathrm{~s} X$ 光电子能谱图

Fig. 9 XPS spectra of N1s in $\mathrm{ZrPTA}$ (a), $\mathrm{ZrPTA} / \mathrm{Pb}^{2+}$ (b), $\mathrm{ZrPTA} / \mathrm{Cd}^{2+}(\mathrm{c})$ and $\mathrm{ZrPTA} / \mathrm{Cu}^{2+}(\mathrm{d})$

不同的吸附方式会导致吸附剂对被吸附物吸附 容量的巨大差异。研究者认为, ZrPTA 对金属离子的
吸附属于物理化学吸附。ZrPTA 骨架结构中含有大 量的 $\mathrm{N}$ 元素和 $\mathrm{C}=\mathrm{O}$ 基团, $\mathrm{C}=\mathrm{O}$ 基团中的 $\mathrm{O}$ 元素以 及 $\mathrm{N}$ 元素最外层轨道上的孤对电子均能够和金属元 素核外空轨道进行配位，从而将金属离子固载于吸 附剂的孔内区域。吸附剂和金属离子间的配位作用 弥补了主客体之间仅靠范德华力作用的局限性，使 其吸附容量大大增加。Z ZrPTA、 ZrPTA $/ \mathrm{Pb}^{2+}$ 、 $\mathrm{ZrPTA} / \mathrm{Cd}^{2+}$ 和 $\mathrm{ZrPTA} / \mathrm{Cu}^{2+}$ 中 $\mathrm{N} 1 \mathrm{~s}$ 光电子能谱如图 $9 \mathrm{a} \sim \mathrm{d}$ 所示。在 ZrPTA 中, $\mathrm{N}$ 元素以 $\mathrm{N}-\mathrm{C}$ 键和 $\mathrm{N}-\mathrm{P}$ 键两种状态存在, $\mathrm{N} 1 \mathrm{~s}$ 结合能分别位于 $400.80\left(\mathrm{~N}_{\mathrm{N}-\mathrm{C}}\right)$ 及 $401.95 \mathrm{eV}\left(\mathrm{N}_{\mathrm{N}-\mathrm{P}}\right)$ 。但在 $\mathrm{ZrPTA} / \mathrm{Pb}^{2+}$ 和 $\mathrm{ZrPTA} / \mathrm{Cd}^{2+}$ 中, $\mathrm{N}$ 元素均以三种状态存在。 $\mathrm{N} 1 \mathrm{~s}$ 在 $\mathrm{ZrPTA} / \mathrm{Pb}^{2+}$ 中的三种结合能位于 $399.98\left(\mathrm{~N}_{\mathrm{N}-\mathrm{C}}\right) 、 401.59\left(\mathrm{~N}_{\mathrm{N}-\mathrm{P}}\right)$ 和 $414.49 \mathrm{eV}\left(\mathrm{N}_{\mathrm{N} \rightarrow \mathrm{Pb} 2+}\right)$ 处; 在 $\mathrm{ZrPTA} / \mathrm{Cd}^{2+}$ 中的三种结合 能分别位于 $400.68\left(\mathrm{~N}_{\mathrm{N}-\mathrm{C}}\right) 、 405.92\left(\mathrm{~N}_{\mathrm{N}-\mathrm{P}}\right)$ 和 $412.71 \mathrm{eV}$ $\left(\mathrm{N}_{\mathrm{N} \rightarrow \mathrm{Cd} 2}\right)$ 。与 $\mathrm{N}-\mathrm{C}$ 键在 ZrPTA 中的 $\mathrm{N} 1 \mathrm{~s}$ 电子结合 能相比, $\mathrm{ZrPTA} / \mathrm{Pb}^{2+}$ 及 $\mathrm{ZrPTA} / \mathrm{Cd}^{2+}$ 中的 $\mathrm{N}_{\mathrm{N}-\mathrm{C}}$ 电子结 合能发生了明显的蓝移现象，说明 $\mathrm{N}$ 元素在上述两 种体系中受到了强烈的作用力, 这种作用力很大程 度上来自其与金属离子的配位作用。ZrPTA 骨架结 构中的 $\mathrm{P}=\mathrm{O}$ 也有可能与金属离子发生配位作用，但 在图 $2 \mathrm{~A}(\mathrm{c})$ (红外光谱中并没有 $\mathrm{P}=\mathrm{O} \rightarrow \mathrm{M}$ 红外吸收 $\left(1276 \mathrm{~cm}^{-1}\right)$ 的存在, 这说明在吸附体中, 这种配位 作用不存在或存在可能性很小。

在 $\mathrm{ZrPTA} / \mathrm{Cu}^{2+}$ 中, $\mathrm{N}$ 元素以两种形态存在(如图 $9 \mathrm{~d}$ 所示), 表明 $\mathrm{Cu}^{2+}$ 离子在 $\mathrm{ZrPTA}$ 中只以物理吸附 形式存在。吸附剂 ZrPTA 对其较大的吸附容量 $(689.81 \mathrm{mg} / \mathrm{g})$ 可能是由于 $\mathrm{Cu}^{2+}$ 的 Jahn-Teller 效应降 低了离子吸附能所致。

系统温度对吸附过程的影响较大。实验研究了 在金属离子初始浓度固定 $\left(c_{0}=900 \mathrm{mg} / \mathrm{L}\right)$ 的情况下， 因环境温度变化所引起的 ZrPTA 对金属离子吸附作 用的影响。如图 10 所示, 在不同温度下吸附剂对三 种金属离子的吸附呈现出不同变化规律。例如, 在 $40 \sim 60^{\circ} \mathrm{C}, \mathrm{ZrPTA}$ 对 $\mathrm{Pb}^{2+}$ 吸附量呈现出上升趋势; 对 $\mathrm{Cd}^{2+}$, 这种吸附量上升趋势出现在 $50 \sim 60^{\circ} \mathrm{C}$ 之间; 


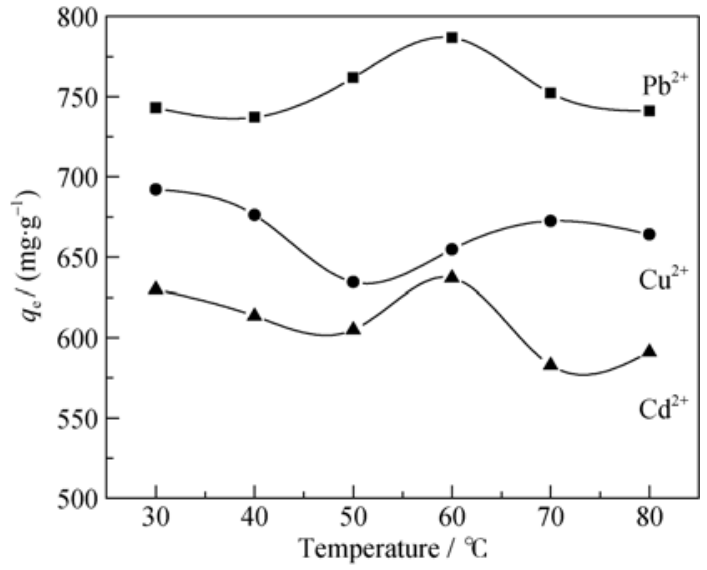

图 10 在固定浓度 $\left(c_{0}=900 \mathrm{mg} / \mathrm{L}\right), \mathrm{ZrPTA}$ 对 $\mathrm{Pb}^{2+} 、 \mathrm{Cu}^{2+}$ 和 $\mathrm{Cd}^{2+}$ 的平衡吸附量随温度 $\left(\right.$ 从 $30^{\circ} \mathrm{C}$ 到 $80^{\circ} \mathrm{C}$ ) 的变化关系曲线

Fig. 10 Relations between the equilibrium adsorption amounts at a given concentration $\left(c_{0}=900 \mathrm{mg} / \mathrm{L}\right)$ and temperature (from $30^{\circ} \mathrm{C}$ to $80^{\circ} \mathrm{C}$ ) of $\mathrm{ZrPTA}$ to $\mathrm{Pb}^{2+}, \mathrm{Cu}^{2+}$ and $\mathrm{Cd}^{2+}$

但对于 $\mathrm{Cu}^{2+}$, 在这一温度区域内 $\left(40 \sim 50^{\circ} \mathrm{C}\right)$, 吸附 剂对其吸附量则呈现下降趋势。但是当系统温度升 至 $60^{\circ} \mathrm{C}$ 以上时, 吸附剂对 $\mathrm{Cd}^{2+}$ 和 $\mathrm{Pb}^{2+}$ 的吸附容量呈 下降趋势, 而对于 $\mathrm{Cu}^{2+}$ 来说, 呈现出上升趋势。对 $\mathrm{Pb}^{2+}$ 和 $\mathrm{Cd}^{2+}$, 最大吸附容量出现在 $60^{\circ} \mathrm{C}$; 而对 $\mathrm{Cu}^{2+}$, 最大吸附容量出现在 $30^{\circ} \mathrm{C}$ 。

溶液的酸碱性能够显著地影响吸附剂的表面性 质以及溶液中金属元素的存在方式。实验研究了 $\mathrm{pH}$ 在 1.0 5.0 范围内 (上述三种金属离子在此 $\mathrm{pH}$ 范围 内不会发生水解而生成 $\mathrm{M}(\mathrm{OH})_{2}$ 沉淀 $)$, 溶液的酸碱 度变化对吸附剂吸附性能的影响。用 $1.0 \mathrm{~mol} / \mathrm{L}$ 的盐 酸溶液调节含金属离子溶液的 $\mathrm{pH}$ 、并使金属离子的 起始浓度为 $900 \times 10^{-6}$, 然后向溶液中加入一定质量 的吸附剂，混合体系搅拌一段时间后，吸附剂对金 属离子的吸附容量与溶液 $\mathrm{pH}$ 对应关系如图 11 所 示。从图 11 可以看出, $\mathrm{pH}$ 在 1.0 4.3 范围, ZrPTA 对

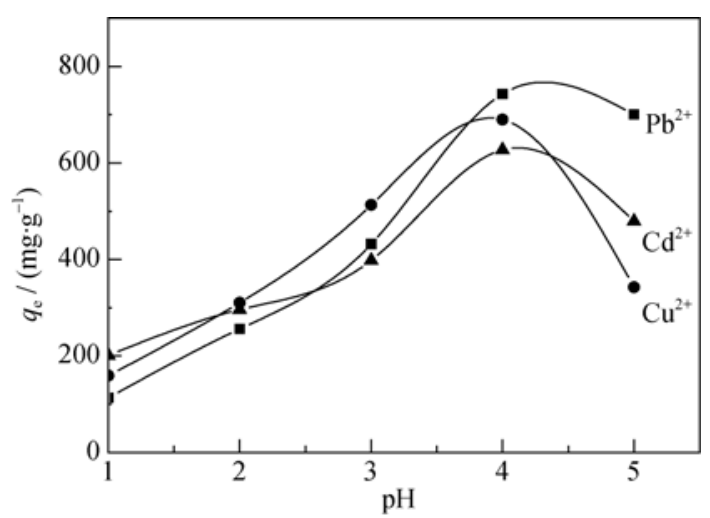

图 11 室温条件下, $\mathrm{ZrPTA}$ 对 $\mathrm{Pb}^{2+} 、 \mathrm{Cu}^{2+}$ 和 $\mathrm{Cd}^{2+}$ 的平衡吸附 量随溶液酸碱度的变化关系曲线

Fig. 11 Equilibrium adsorption amounts of $\mathrm{ZrPTA}$ to $\mathrm{Pb}^{2+}$, $\mathrm{Cu}^{2+}$ and $\mathrm{Cd}^{2+}$ in different $\mathrm{pH}$ solution at room temperature
三种金属离子的吸附容量随溶液的酸度减弱而增 大。在较强的酸性环境中 $(\mathrm{pH}<1.0)$, 吸附剂吸附容量 大幅度减小, 也就是说, 在强酸环境下, 吸附过程 转变为解吸过程。此结果的发现, 为吸附剂的再生 重复利用提供了理论依据。

将吸附组装体置于浓度为 $2.0 \mathrm{~mol} / \mathrm{L}$ 的盐酸溶 液中发现，金属离子几乎全部被解吸，吸附剂可被 完全还原。吸附剂重复利用 12 次, 材料结构依然保 持完好。

\section{3 结论}

在无任何表面活性剂存在条件下，利用双(六 甲撑)三胺, 三氯氧磷，乙酰氯和八水合氧氯化锆水 热法合成得到了一种新型微介孔有机膦酸锆材料 ZrPTA。ZrPTA 具有规整的长条片状微形貌, 材料 内部存在大量的微孔。 $\mathrm{ZrPTA}$ 对 $\mathrm{Pb}^{2+} 、 \mathrm{Cu}^{2+}$ 及 $\mathrm{Cd}^{2+}$ 离子具有良好的吸附性能, 是目前发现的上述三种 离子最好的吸附剂。深入研究发现, 吸附剂对 $\mathrm{Pb}^{2+}$ 和 $\mathrm{Cd}^{2+}$ 的吸附过程是配位吸附及物理吸附共同作用 的结果, 而对 $\mathrm{Cu}^{2+}$ 的吸附仅是物理吸附过程。上述 研究成果为合成更高效吸附剂材料提供了一定的理 论依据。

\section{参考文献:}

[1] EMAMIPOUR H, JOHNSEN D L, ROOD M J, et al. Novel activated carbon fiber cloth filter with functionalized silica nanoparticles for adsorption of toxic industrial chemicals. Adsorption, 2015, 21(4): 265-272.

[2] KRASUCKA P, GOWOREK J, KIERYS A. Synthesis of the mesostructured polymer- silica composite and silicon dioxide through polymer swelling in silica precursor. Adsorption, 2016, 22(4): 663-671.

[3] KRUSZKA B, WIŚNIEWSKI M, TERZYK A P. Phenol adsorption on different nano-sized carbon materials: first comparative study. Adsorption, 2016, 22(4): 437-444.

[4] WU SHENG-JU, LI FENG-TING, XU RAN, et al. Synthesis of thiol-functionalized MCM-41 mesoporous silicas and its application in $\mathrm{Cu}(\mathrm{II}), \mathrm{Pb}(\mathrm{II}), \mathrm{Ag}(\mathrm{I})$ and $\mathrm{Cr}(\mathrm{VI})$ removal. Journal of Nanoparticle Research, 2010, 12(6): 2111-2124.

[5] MERCIER L, PINNAVAIA T J. Heavy metal ion adsorbents formed by the grafting of thiol functionality to mesoporous silica molecular sieves: factors affecting $\mathrm{Hg}^{2+}$ uptake. Environmental Science \& Technology, 1998, 32(18): 2749-2754.

[6] BROWN J, MERCIER L, PINNAVAIA T J. Selective adsorption of $\mathrm{Hg}^{2+}$ by thiol-functionalized nanoporous silica. Chemical Communication, 1999, 69-70.

[7] YUAN Z Y, REN T Z, SU B L. Hierarchically mesostructured titania materials with an unusual interior macroporous structure. Advanced Materials, 2003, 15(17): 1462-1465.

[8] YUAN Z Y, REN T Z, AZIOUNE A, et al. Facile and generalized preparation of hierarchically mesoporous-macroporous binary metal oxide materials. Chemistry of Materials, 2006, 18(7): 1753- 1767. 
[9] ABUTARTOUR A, JIA Y J, LOTfiA E M, et al. A new hierarchical porous zirconium phosphate membrane and its adsorption properties. Microporous and Mesoporous Materials, 2014, 196: 1-7.

[10] ZHANG X J, MA T Y, YUAN Z Y. Synthesis of hierarchically meso-/macroporous titanium tetraphosphonate materials with large adsorption capacity of heavy metal ions. Chemistry Letter, 2008, 37(7): 746-747.

[11] CLEARFIELD A, STYNES J A. The preparation of crystalline zirconium phosphate and some observations on its ion exchange behaviour. Journal of Inorganic Nuclear Chemistry, 1964, 26(1): 117-129.

[12] CLEARFIELD A, BLESSING R H. New crystalline phases of zirconium phosphate possessing ion-exchange properties. Journal of Inorganic Nuclear Chemistry, 1968, 30(8): 2249-2258.

[13] AlBerti G, COSTANTINO U, Allulli S, et al. Crystalline $\mathrm{Zr}\left(\mathrm{RPO}_{3}\right)_{2}$ and $\mathrm{Zr}\left(\mathrm{ROPO}_{3}\right)_{2}$ compounds ( $\mathrm{R}=$ organic radical): a new class of materials having layered structure of the zirconium phosphate type. Journal of Inorganic Nuclear Chemistry, 1978, 40(6): 1113-1117.

[14] CLEARFIELD A. Group IV phosphates as catalysts and catalyst supports. Journal of Molecular Catalysis, 1984, 27(1/2): 251-262.

[15] ALBERTI G. Syntheses, crystalline structure, and ion-exchange properties of insoluble acid salts of tetravalent metals and their salt forms. Accounts of Chemical Research, 1978, 11(4): 163-170.

[16] BELlEZZA F, CIPICIANI A, COSTANTINO U, et al. Adsorp- tion of myoglobin onto porous zirconium phosphate and zirconium benzenephosphonate obtained with template synthesis. Langmuir, 2006, 22(11): 5064-5069.

[17] HOGARTH W H J, DINIZ D C J C, DRENNAN J, et al. Proton conductivity of mesoporous Sol-Gel zirconium phosphates for fuel cell applications. Journal of Materials Chemistry, 2005, 15: $754-758$.

[18] KAPOOR M P, INAGAKI S, YOSHIDA H. Novel zirconium-titanium phosphates mesoporous materials for hydrogen production by photoinduced water splitting. Journal of Physical Chemistry B, 2005, 109(19): 9231-9238.

[19] CRISAFUlly R, MilHOME M A, CAVALCANTE R M, et al. Removal of Some polycyclic aromatic hydrocarbons from petrochemical wastewater using low-cost adsorbents of natural origin. Bioresource Technology, 2008, 99(10): 4515-4519.

[20] REN T Z, YUAN Z Y, SU B L. Surfactant-assisted preparation of hollow microspheres of mesoporous $\mathrm{TiO}_{2}$. Chemical Physics Letter, 2003, 374(1/2): 170-175.

[21] GUERRERO G, MUTIN P, VIOUX A. Anchoring of phosphonate and phosphinate coupling molecules on titania particles. Chemistry of Materials, 2001, 13(11): 4367-4373.

[22] JAIMEZ E, HIX G B, SLADE R C A. Phosphate-phosphonate of titanium (iv) prepared from phosphonomethyliminodiacetic acid: characterisation, n-alkylamine intercalation and proton conductivity. Solid State Ionics, 1997, 97(1-4): 195-201. 